\title{
COMPOSICIÓN CORPORAL Y SOMATOTIPO DE DEPORTISTAS DE ALTO RENDIMIENTO DE LA DISCIPLINA CANOTAJE EN LA REGIÓN DEL BIO BIO, CHILE
}

\author{
BODY COMPOSITION AND SOMATOTYPE ATHLETE OF HIGH PERFORMANCE OF \\ DISCIPLINE BOATING IN THE REGION OF BIO BIO, CHILE
}

\author{
Retamales Muñoz, Francisco(1) \& Valle Parodi, Rodrigo(2)
}

1 Facultad de Ciencias de la Actividad Física, Universidad San Sebastián, Concepción.

2 Departamento de Morfología, Laboratorio de Anatomía, Universidad Andrés Bello, Concepción

RETAMALES MUÑOZ, FRANCISCO \& VALLE PARODI, RODRIGO (2015). Composición corporal y somatotipo de deportistas de alto rendimiento de disciplina canotaje en la regio del Bio Bio, Chile. Mot, Hum 16(2): 61-71.

\section{RESUMEN}

El objetivo del estudio es determinar la Composición Corporal y Somatotipo de deportistas de alto rendimiento de la disciplina canotaje en la región del Bio - Bio que han participado durante el año 2014 de eventos nacionales e internacionales. Muestra: Participaron 14 deportistas de canotaje, divididos en 6 damas (19,32 $\pm 0,88$ años, masa

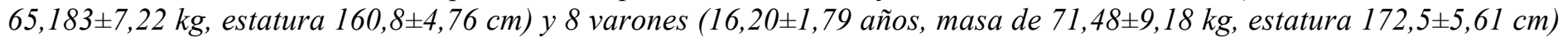
que han sido nominados durante el año 2014 a selectivos nacionales de la especialidad. Metodología: La valoración de la composición corporal se utilizó la fórmula de Kerr (1988), y para el cálculo del somatotipo se utilizó la fórmula de Heath \& Carter (1990), siguiendo las recomendaciones de la sociedad internacional de Kineantropometría (ISAK). Resultados: En cuanto a damas, en la composición corporal se obtuvieron los valores medios de MA 29,15\% - MM 46,27\% - MO 9,74\% - MR 9,86\% - MP 4,97\% con un somatotipo medio de mesoendomorfo y en varones, en composición corporal los valores medios fueron de MA 23,03\% - MM 48,66\% - MO 11,21\% - MR 11,89\% - MP 5,21\% con un somatotipo medio de Mesomorfo balanceado. Conclusiones: Los deportistas de alto rendimiento en la especialidad de canotaje, de la región del Bio Bio, presentan tendencias de mesoendomorfismo relativo en damas, y mesomorfismo en varones, con una tendencia a desviarse de la media ofrecida por el Z-Score o Phantom respecto al modelo pentacompartimental. Con un gran volumen muscular que caracteriza su desarrollo deportivo y diámetros óseos por sobre lo normal.

Palabras Claves: Composición Corporal, Antropometría, Somatotipo, Canotaje

\section{ABSTRACT}

The aim of this study is to determine body composition and somatotype of elite athletes of boating discipline in the region of Bio - Bio who participated in the 2014 national and international events. Sample: 14 athletes participated in boating, divided into 6 ladies $(19,32 \pm 0,88$ years, mass $65,183 \pm 7,22 \mathrm{~kg}$, height $160,8 \pm 4,76 \mathrm{~cm})$ and 8 males $(16,20 \pm 1,79$ years, 9,18 \pm mass of 71,48 kg, height 172,5 $\pm 5,61 \mathrm{~cm})$ who have been nominated for the 2014 national selective specialty. Methodology: The assessment of body composition formula Kerr (1988) and for the calculation of the formula used somatotype Heath \& Carter (1990), following the recommendations of the International Society of Kinanthropometry (ISAK). Results: For ladies, body composition FM mean values were obtained 29.15\% - 46.27\% MM OM 9,74\% - 9,86\% RM - SM 4,97\% with a mean somatotype of mesoendomorphy (4,9 - 5,6 - 0,9) and in males, body composition mean values were 23,03\% FM - MM 48,66\% - 11,21\% MO - RM 11,89 \% - SM 5,21\% with a mean somatotype Mesomorph balanced (2,8 - 5,4 - 1,8). Conclusions: The high performance athletes specializing in boating, Bio Bio region, present trends relative mesoendomorphy ladies, and mesomorphy in males, with a tendency to deviate from the average offered by the Z-Score or phantom relative to pentacompartimental model. With a large muscle volume that characterizes his athletic development and bone diameters above normal.

Keywords: Body composition, anthropometry, somatotype, Kayaking. 


\section{INTRODUCCIÓN}

La disciplina de canotaje, involucra una demanda energética y prestación mecánica asociada a componentes físicos, coordinativos $\mathrm{y}$ volitivos que permiten establecer diferencias notorias en las marcas obtenidas (Cermak, Kuta, \& Parizkova, 1975). En ese entorno la composición corporal y somatotipo es un punto necesario de control para el correcto desarrollo de las metodologías de entrenamiento que involucren la búsqueda de un rendimiento elevado a nivel deportivo (Fry \& Morton, 1991).

El canotaje es un deporte que implica el traslado de un individuo sobre una embarcación ligera y de baja estabilidad en aguas tranquilas, siendo el componente esencial la velocidad de traslación en línea recta en distancias de $200 \mathrm{~m}, 500 \mathrm{~m}, 1000 \mathrm{~m}$, $2000 \mathrm{~m}$ y maratón. Tiene modalidades Canoa y Kayak, pudiendo participar simultáneamente en la misma embarcación de 1 a 4 deportistas.

En Chile, el canotaje se practica esencialmente en la zona centro - sur del país, debido a la existencia de lagunas y ríos aptos para el desarrollo de la especialidad deportiva, destacando la región del Bio - Bio, por sus características geográficas privilegiadas, permite tener polos de desarrollo de la especialidad y por ende deportistas destacados; gracias a la alta presencia de lagunas naturales aptas para el desarrollo de la especialidad deportiva de Canoa y Kayak, teniendo referentes importantes en las comunas de Laja, Quillón, Santa Juana y San Pedro de la Paz. Sumado a ello el aporte que hace el Instituto Nacional de Deportes, a través del programa Centro de Entrenamiento Regional (CER), el cual tiene a la disciplina consignada como "deporte estratégico", sumando esfuerzos para mantener la presencia de los deportistas en los selectivos nacionales.

En el canotaje muchos autores han descrito sus características morfofuncionales para detectar los talentos deportivos que brinden la mejor prestación en las diversas distancias de competencia y modalidad (Bishop, 2000; Misigoj-Duraković \& Heimer, 1992; Van Someren \& Howatson, 2008), es por ello que el estudio de la composición corporal y somatotipo se vuelve crítico al momento de la búsqueda del rendimiento deportivo en competencias de alto nivel (Forbes, Fuller, Krentz, Little, \& Chilibeck, 2009), situación que no es ajena a la realidad del país ya que involucra el estudio de esta población deportiva, objetivo que en los últimos años se destaca con la finalidad de orientar hacia el logro los esfuerzos de los cultores de este deporte (Retamales, 2011).

Es por ello que el presente estudio de composición corporal y somatotipo permitirá identificar de forma concisa la caracterización de los deportistas de canotaje de la región del Bio Bio, en función de sus potencialidades naturales. Debido a esto, es que se permite la visualización de forma evolucionada sobre las alternativas de elección y trabajo diferenciado en el deporte de élite de los últimos años en el extranjero (Ackland, Ong, Kerr, \& Ridge, 2003; Aladro, Machado, \& Bueno, 2007; Carrasco, Martínez, \& Nadal, 2005; Gonzalvo \& Díaz, 2007). A consecuencia de ello, se ha hecho preponderante el contar con información funcional y morfológica clave para el desarrollo de programas efectivos en la búsqueda del máximo rendimiento deportivo (Alfonso, 2010), como así mismo una secularización de la evolución de los deportistas (Carvajal et al., 2011); esto ha de tener consecuencias concretas en la incorporación de nuevos deportistas gracias a sus condicionantes particulares (Hoffman, 2006), (Maud \& Foster, 2006), las cuales para esta investigación proporciona elementos concretos para futuras elecciones e incorporaciones de nuevos talentos deportivos, con expectativas de desarrollo acorde a la realidad de la especialidad deportiva.

El objetivo del estudio es determinar la Composición Corporal y Somatotipo de deportistas de alto rendimiento en la disciplina canotaje de la región del Bio - Bio que han participado durante el año 2014 de selectivos nacionales.

\section{METODOLOGÍA}

El presente estudio es de tipo descriptivo y transversal; para su realización se contó con la participación de 6 deportistas damas $(18,97 \pm 0,34$ 
años, masa $65,18 \pm 7,22 \mathrm{~kg}$, estatura $160,8 \pm 4,76$ $\mathrm{cm})$ y 8 varones $(16,20 \pm 1,79$ años, masa de $71,48 \pm 9,18 \mathrm{~kg}$, estatura $172,5 \pm 5,61 \mathrm{~cm}$ ), que durante el año 2014 han participado de eventos nacionales e internacionales en la especialidad y que pertenecen a la región del Bio - Bio. Todos los sujetos fueron informados y firmaron su consentimiento autorizando las evaluaciones.

La valoración antropométrica de los deportistas se realizó siguiendo el protocolo ISAK (International Society for Advancement in Kinanthropometry) de perfil restringido contenido en el Manual respectivo (Marfell-Jones, Stewart, \& de Ridder, 2012). Las mediciones se realizaron en el mismo lugar y durante un único día por cada grupo. Se realizó un perfil completo de cada deportista. Este comprende la estatura y el peso más pliegues cutáneos (tríceps, subescapular, bíceps, cresta ilíaca, supraespinal, abdominal, muslo medio, pantorrilla medial), perímetros (brazo relajado y contraído, antebrazo máximo, muñeca, tórax, cintura, cadera, muslo máximo, muslo medio, pantorrilla máximo), diámetros (húmero y fémur). Todas las mediciones fueron tomadas en el lado derecho del cuerpo y todos los deportistas se encontraban en periodo precompetitivo.

Los datos se procesaron y analizaron en el Software de análisis de datos antropométricos - Antropogym 2.0 de autoría de Francis Holway antropometrista ISAK nivel 4; la cual se encuentra basada en el protocolo de la International Society for the Advancement of Kinathrophometry (ISAK) el cual establece cuatro (4) factores de valoración, a saber: Composición corporal (Ross \& Kerr, 1991), Puntuación de proporcionalidad Phantom (Shephard et al., 1985), Estimación de gasto energético (Harris \& Benedict, 1918), (FAO, 1985) y (Frankenfield, Muth, \& Rowe, 1998); y Somatotipo (Carter \& Heath, 1990).

En el caso del evaluador, fueron sometidos al protocolo de test - retest para puntuar su grado de error intramuestras, quedando determinado en 1,49 $\%$ y $1,50 \%$ respectivamente. Por último, se realizó estadística descriptiva para determinar medias, desviaciones estándar, mínimos y máximos de las características, y los resultados obtenidos por los jugadores, con el propósito de describir la composición corporal y el somatotipo de los deportistas.

Obtenidos los datos correspondientes, se procesan estos en planilla de cálculo Excel (Microsoft, 2010), para la obtención de las coordenadas, para la determinación del somatotipo y de las ecuaciones que determinan la composición corporal. Además de procesar la información mediante el software SPSS versión 20, para el desarrollo de la estadística del estudio.

\section{RESULTADOS}

Realizadas las mediciones en ambos grupos (damas y varones), se pudo establecer las medias de cada uno (Tabla 1 y Tabla 2), en lo que respecta a las curvas de crecimiento normal, se puede observar que estas medias no difieren significativamente a los procesos normales de desarrollo de jóvenes dentro del mismo intervalo de edad (16 - 19 años), estando ambos por debajo del p50 (percentil 50), pero sobre el p45 (percentil 45) establecido en el patrón publicado por la Organización Mundial para la Salud (Onis et al., 2007). Lo cual hace presuponer que la población de deportistas de canotaje se mantiene en rangos normales para su edad. Ante ello si se logra determinar una diferencia de $-2,3 \mathrm{~cm}$ de altura y $+3,64$ puntos de Índice de Masa Corporal (IMC) respecto la media en damas; y $-1,1 \mathrm{~cm}$ de altura, $\mathrm{y}+3,57$ puntos de IMC sobre la media en varones, ambos en relación al p50 descrito previamente.

A su vez, y comparando los segmentos corporales de los deportistas entre ellos se puede observar una diferencia de un $0,90 \%$ en damas y $0,88 \%$ en varones, lo cual indica que su desarrollo a la vez se va encaminando en favor de la prueba en la que compite (Aragonés \& Casajús, 1991), lo que afirma el hecho de que una modificación funcional del organismo ante un esfuerzo particular (Delgado, 2010; Tschiene, 1996). 
RETAMALES MUÑOZ, FRANCISCO \& VALLE PARODI, RODRIGO (2015). Composición corporal y somatotipo de deportistas de alto rendimiento de disciplina canotaje en la regio del Bio Bio, Chile. Mot, Hum 16 (2): 61-71.

Bajo esta indicación se toma como referencia de modificación el stress deportivo y físico sufrido por el cuerpo en los entrenamientos y que los condiciona favorablemente hacia el rendimiento (Claessens, Lefevre, Beunen, \& Malina, 2000; Malina, 2006).

Tabla 1: Descriptivos de muestras en damas respecto a diámetros, perímetros y pliegues valorados

\begin{tabular}{|c|c|c|c|c|}
\hline \multicolumn{5}{|c|}{ Estadística Descriptiva damas } \\
\hline & Mínimo & Máximo & Media & Desv. típ. \\
\hline Edad & 18,43 & 21,00 & 19,32 & 0,88 \\
\hline Peso & 57,90 & 78,70 & 65,183 & 8,25 \\
\hline Estatura & 153,80 & 169,00 & 160,883 & 5,61 \\
\hline Talla Sentado & 83,50 & 88,00 & 85,467 & 1,84 \\
\hline \multicolumn{5}{|c|}{ DIAMETROS } \\
\hline Biacromial & 34,70 & 40,60 & 37,383 & 2,28 \\
\hline Tórax Transverso & 24,50 & 32,10 & 27,000 & 3,03 \\
\hline Tórax Anteroposterior & 15,70 & 22,30 & 17,933 & 2,71 \\
\hline Bi-Ilicrestideo & 16,60 & 29,70 & 26,217 & 5,21 \\
\hline Humeral & 6,00 & 6,50 & 6,250 & 0,21 \\
\hline Femoral & 8,80 & 9,90 & 9,367 & 0,44 \\
\hline \multicolumn{5}{|c|}{ PERIMETROS } \\
\hline Cabeza & 53,00 & 56,00 & 54,733 & 1,32 \\
\hline Brazo Relajado & 27,60 & 35,00 & 30,767 & 2,93 \\
\hline Brazo Contraído & 27,20 & 35,00 & 30,967 & 2,99 \\
\hline Antebrazo Máximo & 23,00 & 28,40 & 25,483 & 2,04 \\
\hline Tórax Mesoesternal & 92,00 & 113,60 & 98,567 & 8,69 \\
\hline Cintura (Mínima) & 71,40 & 93,70 & 77,150 & 8,98 \\
\hline Cadera (Máxima) & 91,80 & 109,50 & 99,200 & 6,95 \\
\hline Muslo Máximo & 51,40 & 64,70 & 59,100 & 4,79 \\
\hline Muslo Medial & 47,00 & 56,60 & 53,417 & 3,74 \\
\hline Pantorrilla & 33,70 & 39,00 & 36,550 & 2,03 \\
\hline \multicolumn{5}{|c|}{ PLIEGUES } \\
\hline Tríceps & 13,00 & 21,00 & 17,33 & 3,19 \\
\hline Subescapular & 7,00 & 27,00 & 12,50 & 8,01 \\
\hline Supraespinal & 7,00 & 27,00 & 16,50 & 7,42 \\
\hline Abdominal & 14,00 & 32,00 & 24,00 & 7,40 \\
\hline Muslo Medial & 9,00 & 26,50 & 19,10 & 6,56 \\
\hline Pantorrilla & 10,00 & 19,00 & 14,50 & 3,77 \\
\hline Suma de 6 Pliegues & 60,00 & 152,50 & 103,93 & \\
\hline
\end{tabular}

En cuanto a la composición corporal se obtuvieron los valores medios en damas de MA (masa adiposa) 29,15\% - MM (masa muscular) 46,27\% - MO (masa ósea) 9,74\% - MR (masa residual) 9,86\% MP (masa piel) 4,97\%. Y en varones, MA (masa adiposa) 23,03\% - MM (masa muscular) 48,66\% MO (masa ósea) 11,21\% - MR (masa residual)
$11,89 \%$ - MP (masa piel) 5,21\%. Lo cual destaca en ambos sexos la masa muscular que se mantiene cercana al $50 \%$ del total corporal, esto principalmente por las características propias del deporte. En la siguiente tabla $\left(\mathrm{N}^{\circ} 3\right)$, se expresan los resultados de composición Corporal de cada grupo de la muestra. 
RETAMALES MUÑOZ, FRANCISCO \& VALLE PARODI, RODRIGO (2015). Composición corporal y somatotipo de deportistas de alto rendimiento de disciplina canotaje en la regio del Bio Bio, Chile. Mot, Hum 16 (2): 61-71.

Tabla 2: descriptivos de la muestras en varones, respecto a diámetros, perímetros y pliegues valorados

\begin{tabular}{|c|c|c|c|c|}
\hline \multicolumn{5}{|c|}{ Estadística Descriptiva varones } \\
\hline & Mínimo & Máximo & Media & Desv. típ. \\
\hline Edad & 16,20 & 18,70 & 16,68 & 1,79 \\
\hline Peso & 61,10 & 86,80 & 71,475 & 9,18 \\
\hline Estatura & 167,00 & 179,20 & 172,513 & 5,61 \\
\hline Talla Sentado & 86,20 & 94,00 & 89,438 & 2,74 \\
\hline \multicolumn{5}{|c|}{ DIAMETROS } \\
\hline Biacromial & 38,30 & 43,20 & 40,875 & 1,63 \\
\hline Tórax Transverso & 26,80 & 33,10 & 28,713 & 2,05 \\
\hline Tórax Anteroposterior & 15,60 & 22,70 & 19,625 & 3,02 \\
\hline Bi-Ilicrestideo & 23,90 & 29,40 & 26,575 & 1,88 \\
\hline Humeral & 6,30 & 7,40 & 6,813 & 0,36 \\
\hline Femoral & 9,20 & 10,10 & 9,613 & 0,30 \\
\hline \multicolumn{5}{|c|}{ PERIMETROS } \\
\hline Cabeza & 55,00 & 57,30 & 56,075 & 0,74 \\
\hline Brazo Relajado & 25,50 & 35,50 & 30,675 & 3,61 \\
\hline Brazo Contraído & 29,00 & 37,10 & 32,925 & 2,69 \\
\hline Antebrazo Máximo & 25,40 & 29,30 & 27,400 & 1,40 \\
\hline Tórax Mesoesternal & 90,30 & 104,60 & 97,538 & 5,90 \\
\hline Cintura (Mínima) & 73,50 & 87,00 & 80,375 & 4,93 \\
\hline Cadera (Máxima) & 86,00 & 103,00 & 92,563 & 5,94 \\
\hline Muslo Máximo & 50,40 & 58,60 & 54,300 & 3,07 \\
\hline Muslo Medial & 47,00 & 56,00 & 51,250 & 3,26 \\
\hline Pantorrilla & 33,50 & 40,20 & 36,863 & 2,36 \\
\hline \multicolumn{5}{|c|}{ PLIEGUES } \\
\hline Tríceps & 5,00 & 13,00 & 8,500 & 2,82 \\
\hline Subescapular & 5,00 & 16,00 & 9,688 & 3,78 \\
\hline Supraespinal & 4,00 & 21,00 & 10,000 & 6,18 \\
\hline Abdominal & 5,50 & 25,00 & 13,938 & 7,92 \\
\hline Muslo Medial & 5,00 & 20,50 & 11,063 & 5,05 \\
\hline Pantorrilla & 4,00 & 12,50 & 8,063 & 2,97 \\
\hline Suma de 6 Pliegues & 28,50 & 108,00 & 61,25 & \\
\hline
\end{tabular}

Tabla 3: Resultados de la composición corporal por Género de deportistas

\section{Resultados Composición Corporal}

\begin{tabular}{cccccc} 
& \%MA & \%MM & \%MO & \% MR & \%MP \\
\hline $\begin{array}{c}\text { Damas } \\
(\mathbf{n}=\mathbf{6})\end{array}$ & $29,15 \pm 1,71$ & $46,27 \pm 2,71$ & $9,74 \pm 0,58$ & $9,86 \pm 0,57$ & $4,97 \pm 0,29$ \\
$\begin{array}{c}\text { Varones } \\
(\mathbf{n}=\mathbf{8})\end{array}$ & $23,03 \pm 0,40$ & $48,66 \pm 0,84$ & $11,21 \pm 0,93$ & $11,89 \pm 0,21$ & $5,21 \pm 0,09$
\end{tabular}

Leyenda: $M A=$ Masa Adiposa $; M M=$ Masa Muscular, $M O=$ Masa Ósea $;$ MR = Masa Residual $;$ MP = Masa Piel 
RETAMALES MUÑOZ, FRANCISCO \& VALLE PARODI, RODRIGO (2015). Composición corporal y somatotipo de deportistas de alto rendimiento de disciplina canotaje en la regio del Bio Bio, Chile. Mot, Hum 16 (2): 61-71.

En cuanto al Somatotipo de Heath-Carter se obtuvo un Somatotipo medio de $(4,9-5,6-0,9)$ y su clasificación es "Meso - endomorfo" en damas, y en varones de $(2,8-5,4-1,8)$ clasificándolos en "Mesomorfo balanceado", como se indica en la Tabla ${ }^{\circ} 4$. Al integrar los datos en la somatocarta, se aprecia el posicionamiento relativo (medias por grupos) que demuestras las desviaciones respecto a la tendencia ideal descrito por diversos estudios referidos a la especialidad (Gráfico $\left.{ }^{\circ} 1\right)$.

Figura 1: Somatocartas de los deportistas disgregados por Género, indicando posicionamiento actual (circulo azul) y posicionamiento presentado por deportistas olímpicos de la especialidad (rombo rojo) en Ackland et al. (2003), Morphological characteristics of Olympic sprint canoe and kayak paddlers. Journal of Science and Medicine in Sport, 6(3), 285-294.

\section{Somatocartas}

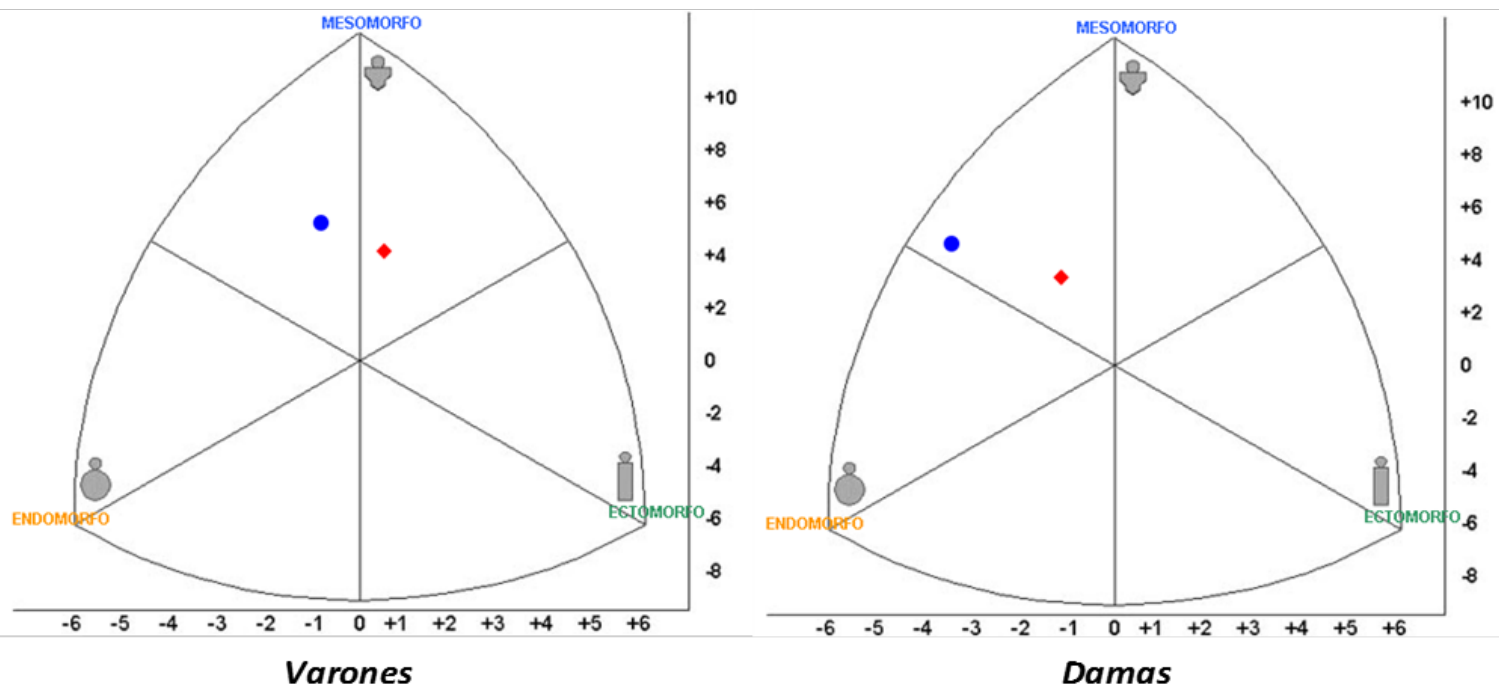

Tabla 4: Somatotipo promedio y distribución de componentes de los deportistas evaluados, de acuerdo a su Género.

Resultados Somatotipo

\begin{tabular}{ccccc}
\cline { 2 - 4 } & ENDO & MESO & ECTO & CLASIFICACIÓN \\
\hline $\begin{array}{c}\text { Damas } \\
(\mathbf{n}=\mathbf{6})\end{array}$ & $4,9 \pm 0,58$ & $5,6 \pm 1,57$ & $0,9 \pm 0,07$ & $\begin{array}{c}\text { Meso } \\
\text { endomorfismo }\end{array}$ \\
$\begin{array}{c}\text { Varones } \\
(\mathbf{n}=\mathbf{8})\end{array}$ & $2,8 \pm 0,67$ & $5,4 \pm 0,46$ & $1,8 \pm 0,44$ & $\begin{array}{c}\text { Mesomorfo } \\
\text { Balanceado }\end{array}$ \\
\end{tabular}


RETAMALES MUÑOZ, FRANCISCO \& VALLE PARODI, RODRIGO (2015). Composición corporal y somatotipo de deportistas de alto rendimiento de disciplina canotaje en la regio del Bio Bio, Chile. Mot, Hum 16 (2): 61-71.

Respecto a la puntuación del Z-Score se logra identificar que los perímetros en las damas (gráfico $\mathrm{n}^{\circ} 2$ ) indican valores de masa muscular por sobre lo esperado relacionándose de manera positiva por sobre la media estimativa, asumiendo esto y respecto a la composición corporal, vemos que existe una concordancia de esa diferencia $( \pm 2,3$ puntos porcentuales), asumiendo el mayor desarrollo muscular propio de la especialidad deportiva.

Es necesario mencionar las características que nos arroja el promedio de la talla sentado, considerando que la media del Z-Score está basada en una población extremadamente heterogénea (Dibley, Staehling, Nieburg, \& Trowbridge, 1987). Encontrándose el valor de las damas bajo el promedio ya mencionado, afectando en forma clara los aspectos deportivos propios de la especialidad, esto asumiendo los largos de palanca y las fuerzas que se ven implicadas en el gesto técnico de la remada.

Los pliegues cutáneos demuestran un porcentaje de grasa elevado arrojando valores fuera del rango ideal que se sitúa entre -2 y -3 puntos. No encontrándose ningún pliegue dentro de la media propuesta.

Figura 2: Score-Z de muestra Damas

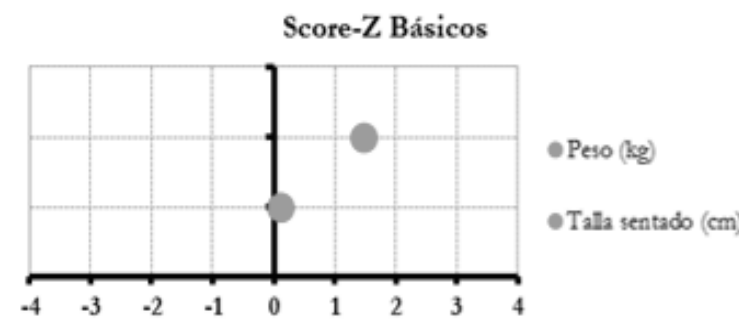

Score-Z Perímetros

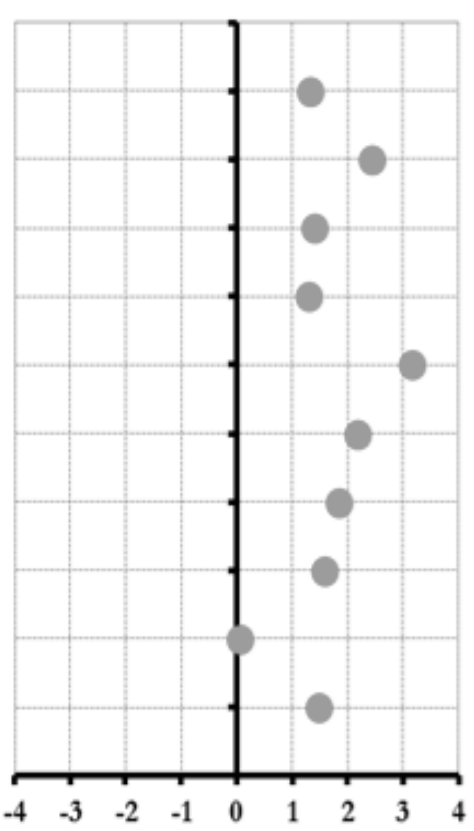

- Brazo Relajado

- Brazo Flexionado en Tensión

- Antebeazo

- Tómax Mesoestemal

- Cintura (mínima)

- Cadens (mixima)

- Muslo (supecior)

- Muslo (medal)

- Pantorilla (maxima)
Score-Z Diámetros

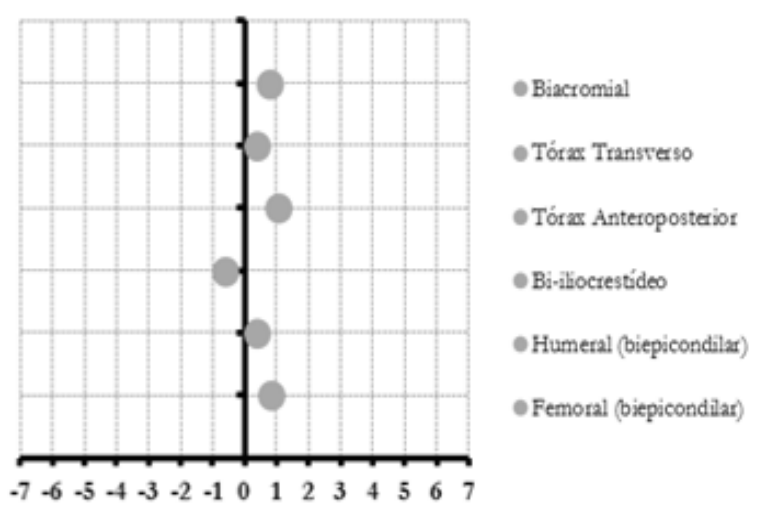

$\begin{array}{lllllllllllllll}-7 & -6 & -5 & -4 & -3 & -2 & -1 & 0 & 1 & 2 & 3 & 4 & 5 & 6 & 7\end{array}$

\section{Score-Z Pliegues}

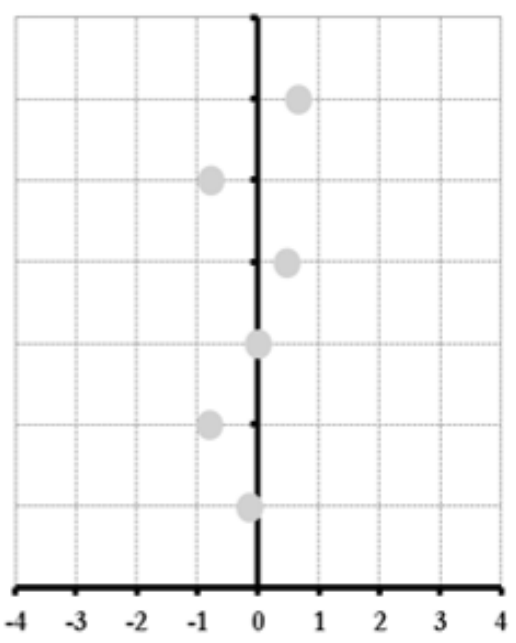

- Triceps

- Subescapular

- Suprespinal

- Abdominal

- Muslo (medal)

- Pantorilla 
RETAMALES MUÑOZ, FRANCISCO \& VALLE PARODI, RODRIGO (2015). Composición corporal y somatotipo de deportistas de alto rendimiento de disciplina canotaje en la regio del Bio Bio, Chile. Mot, Hum 16 (2): 61-71.

Gráfico 3: Score-Z de muestra Varones
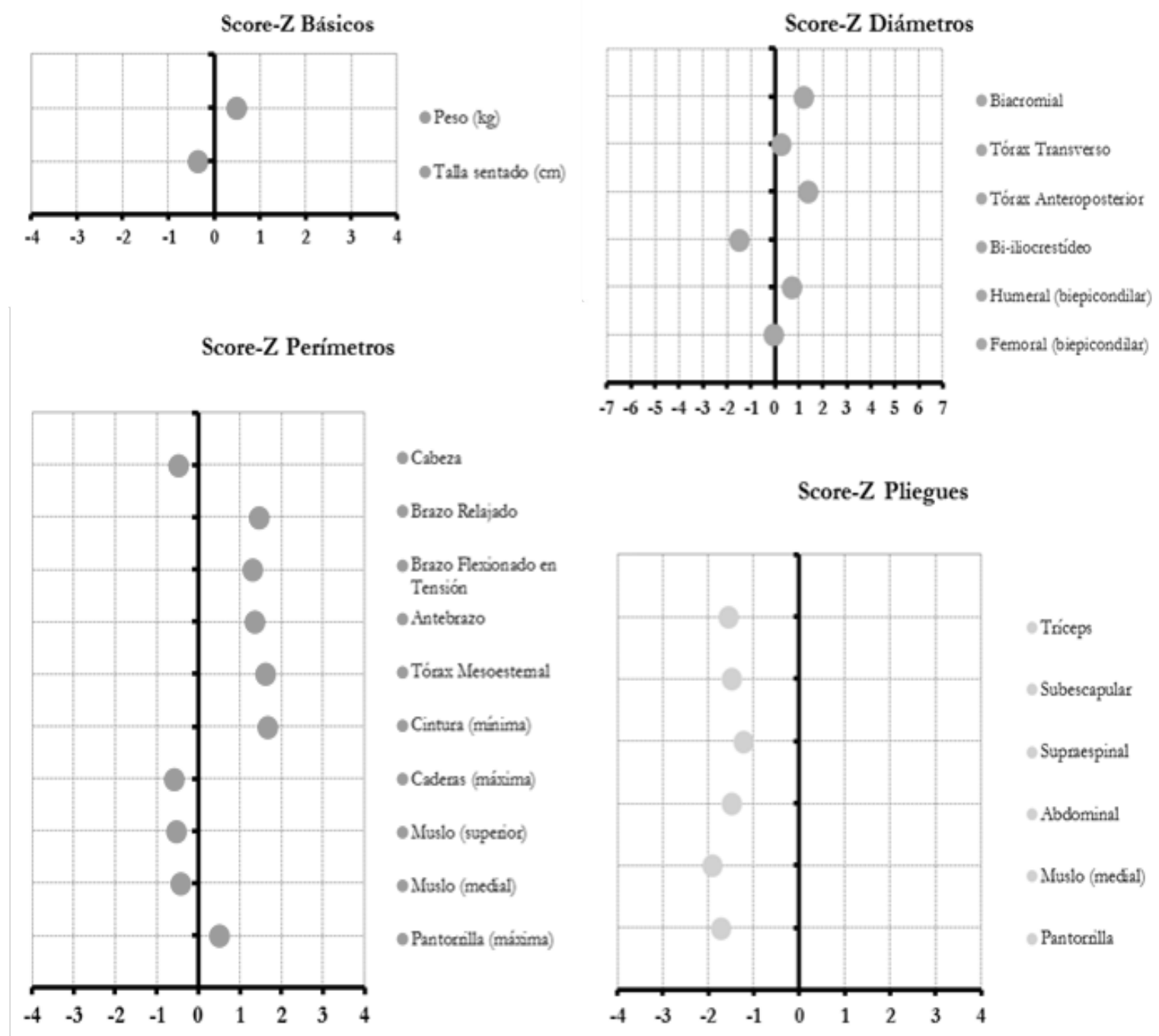

\section{Score-Z Pliegues}

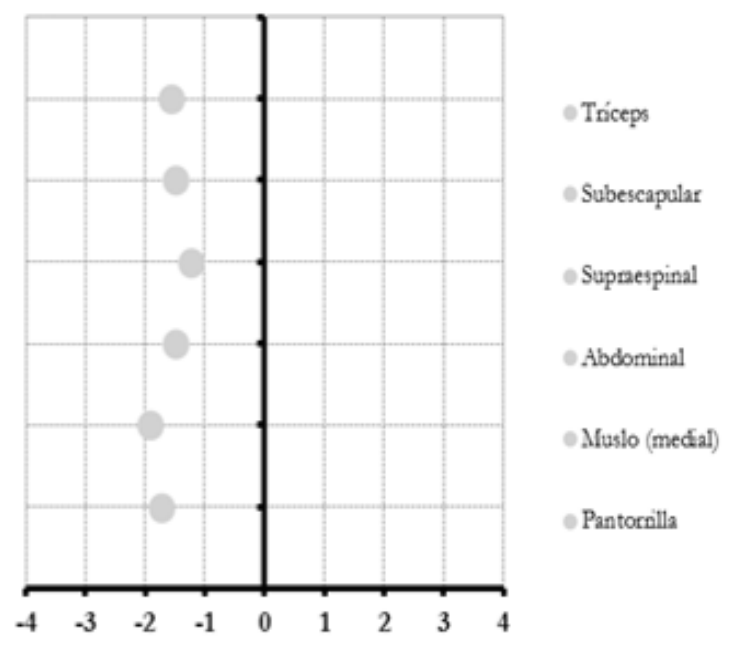

En el gráfico $n^{\circ} 3$ referente a los puntajes Z-Score de varones, observando los pliegues cutaneos podemos observar dentro de la normalidad todos los pliegues evaluados, distinta situación tenemos en relación a los perímetros del tronco superior donde los indices de masa muscular son ideales siendo correspondientes al trabajo muscular del deporte en particular, como contraparte se observa una disminución del IMC, pudiendose observar como

contraparte del trabajo muscular analizado en el tren superior.

Analizando los Z-Score básicos, nuevamente nos encontramos con el aspecto deficitario en la talla sentado marcando una tónica entre las damas y varones en relación a este estudio en particular. 


\section{DISCUSIÓN Y CONCLUSIONES}

El análisis de parámetros antropométricos básicos, composición corporal y somatotipo en deportistas de alto rendimiento en la disciplina de canotaje, permite que se puedan brindar nuevas expectativas de programación del entrenamiento, incremento de variables para la búsqueda $\mathrm{y}$ detección de talentos deportivos que respondan a este perfil descrito (Sosa, 2006). Es así como Ackland et al. (2003) verifica que la composición corporal en deportistas de canotaje se ve alterada en relación a porcentajes de masa muscular (MM), encontrando valores medios superiores respecto a la población normal. En otro estudio refleja bajo la misma premisa que la relación de MM se asume en función al somatotipo del individuo y no solamente al tipo de entrenamiento que recibe (Espinoza, 2010).

Atendiendo a estas diferencias, es que se verifica que los deportistas de élite en canotaje constituyen ciertas similitudes respecto a su proporcionalidad ya que al presentar diferencias entre sus segmentos corporales, siendo reducidos en las extremidades, generan un mayor poder de tracción al momento de efectuar la remada, situación que se repite en varios estudios hechos previamente (Ackland et al., 2003; Alacid, Muyor, \& López-Miñarro, 2011; Carrasco et al., 2005).

Esto evidencia que la distribución de las proporcionalidades y de la composición corporal tiene efecto directo en la distribución somatotípica a través del tiempo (Carvajal et al., 2011), la cual tiende a establecerse en damas dentro de rangos medios del mesoendomorfismo y en varones el mesomorfismo balanceado. Se debe atender que al considerarse solamente las condiciones de composición corporal y somatotipo sin mediar en el tipo de prueba, se deja abierta la puerta para variaciones sutiles que involucren mejoras en la prestación deportiva en su fase mecánica como fisiológica.

Acorde a los datos obtenidos, y el procesamiento de estos, se puede concluir que los individuos pertenecientes a la disciplina de canotaje y que se encuentran dentro de la categoría de Alto Rendimiento en el género damas, según los resultados del somatotipo presentan moderada adiposidad relativa, situándolas en un somatotipo de tipo meso- endomórfico; con grasa subcutánea que cubre los contornos musculares y óseos; una apariencia más blanda, y moderado desarrollo músculo -esquelético relativo. Con palancas segmentarias reducidas respecto a la media, y bajo estándares de desarrollo normales para individuos de su edad.

A razón de esto, marca un biotipo mesomorfo predominante con un porcentaje medio alto en el componente graso, pudiendo con ello perjudicar la dinámica sobre el agua y con ello su perfomance deportiva tomando en cuenta que el canotaje es un deporte en donde los aspectos de peso, alcance segmentario, palancas y tracciones son fundamentales en el rendimiento final.

En relación a los varones nos encontramos con características antropométricas que nos indican una baja adiposidad relativa; poca grasa subcutánea; contornos musculares y óseos visibles. Moderado desarrollo músculo esquelético relativo; mayor volumen muscular y huesos y articulaciones de mayores dimensiones. Gran volumen por unidad de altura; extremidades relativamente voluminosas. De la misma manera se posicionan más cercanos al ideal tomando en consideración los valores de la somatocarta que nos entrega valores referenciados respecto a deportistas olímpicos de la especialidad (Ackland et al., 2003).

El aporte de estos datos puede ser de referencia concreta para futuros procesos de selección de deportistas en la especialidad de canotaje, tomando como referencia valores antropométricos contemporáneos de la realidad de la región y lo que se puede caracterizar como ideal. Entendiendo este tipo de procesos como necesarios para establecer una tendencia secular que concrete un patrón de composición corporal y somatotipológico en los procesos de formación hacia el nivel deportivo que se pretende establecer en la región y de la misma manera con las demás regiones que presenten la especialidad deportiva y en donde se busque el rendimiento. 


\section{AGRADECIMIENTOS}

Se agradece la colaboración de los Técnicos y Deportistas pertenecientes al Centro de Entrenamiento Regional (CER), y al Instituto Nacional de Deportes - región del Bio Bio por facilitar los datos para el presente estudio.

\section{REFERENCIAS BIBLIOGRÁFICAS}

Ackland, T., Ong, K., Kerr, D., \& Ridge, B. (2003). Morphological characteristics of Olympic sprint canoe and kayak paddlers. Journal of Science and Medicine in Sport, 6(3), 285-294.

Alacid, F., Muyor, J., \& López-Miñarro, P. (2011). Perfil antropométrico del canoísta joven de aguas tranquilas. International Journal of Morphology, 29(3), 835-840.

Aladro, A., Machado, M., \& Bueno, E. (2007). Somatotipo de piragüistas élite de Cuba. Revista en Ciencias del Movimiento Humano y Salud, 4(2), 115.

Alfonso, J. (2010). Somatotipo de nadadores juveniles de nivel competitivo internacional. Avanzada Científica, 4(1).

Aragonés, M., \& Casajús, J. (1991). Modificaciones antropométricas debidas al entrenamiento. Estudios longitudinales. Archivos de Medicina del Deporte, 8(32), 345 .

Bishop, D. (2000). Physiological predictors of flatwater kayak performance in women. European Journal of Applied Physiology, 82(1-2), 91-97.

Carrasco, L., Martínez, E., \& Nadal, C. (2005). Perfil antropométrico, somatotipo y composición corporal de jóvenes piragüistas International Journal of Medicine and Science of Physical Activity and Sport, 5(20), 270-281.

Carter, J., \& Heath, B. (1990). Somatotyping: development and applications (Vol. 5): Cambridge University Press.
Carvajal, W., Espinosa, A., López, A., Rodriguez, D., Martinez, M., \& Echavarría, I. (2011). Evolución morfológica en atletas cubanos masculinos de canoa y kayak de alto rendimiento: período 1980-2010. Rev. Cub. Med. Dep \& Cul. Fis., 6(2).

Cermak, J., Kuta, I., \& Parizkova, J. (1975). Some predispositions for top performance in speed canoeing and their changes during the whole year training program. The Journal of sports medicine and physical fitness, 15(3), 243.

Claessens, A., Lefevre, J., Beunen, G., \& Malina, R. (2000). The contribution of anthropometric characteristics to performance scores in elite female gymnasts. The Journal of sports medicine and physical fitness(39), 355-360.

Delgado, M. (2010). Fundamentación anatómico funcional del rendimiento y del entrenamiento de la resistencia del niño y del adolescente. Motricidad. European Journal of Human Movement, 1.

Dibley, M., Staehling, N., Nieburg, P., \& Trowbridge, F. (1987). Interpretation of Z-score anthropometric indicators derived from the international growth reference. The American journal of clinical nutrition, 46(5), 749-762.

Espinoza, A. (2010). Aceleración secular de variables morfológicas en deportistas de canotaje cubanos. Periodo 1980-2010. (Master en control médico del entrenamiento deportivo), Instituto Superior de Ciencias Médicas de la Habana, La Habana, Cuba.

FAO, O. (1985, 07/10/1985). Necesidades de energía $y$ de proteinas. Paper presented at the Reunión Consultiva Conjunta FAO/OMS/UNU de Expertos, Ginebra, Suiza.

Forbes, S., Fuller, D., Krentz, J., Little, J., \& Chilibeck, P. (2009). Anthropometric and physiological predictors of flat-water $1000 \mathrm{~m}$ kayak performance in young adolescents and the effectiveness of a high volume training camp. International Journal of Exercise Science, 2(2), 106-114. 
RETAMALES MUÑOZ, FRANCISCO \& VALLE PARODI, RODRIGO (2015). Composición corporal y somatotipo de deportistas de alto rendimiento de disciplina canotaje en la regio del Bio Bio, Chile. Mot, Hum 16 (2): 61-71.

Frankenfield, D., Muth, E., \& Rowe, W. (1998). The Harris-Benedict studies of human basal metabolism: history and limitations. Journal of the American Dietetic Association, 98(4), 439445. doi: 10.1016/S0002-8223(98)00100-X

Fry, R., \& Morton, A. (1991). Physiological and kinanthropometric attributes of elite flatwater kayakists. Medicine and science in sports and exercise, 23(11), 1297-1301.

Gonzalvo, A., \& Díaz, M. (2007). Somatotipo en piragüistas élite de Cuba. MHSalud: Movimiento Humano y Salud, 4(2), 3.

Harris, J., \& Benedict, F. (1918). A biometric study of human basal metabolism. Proceedings of the National Academy of Sciences of the United States of America, 4(12), 370.

Hoffman, J. (2006). Norms for fitness, performance, and health: Human Kinetics Champaign, IL.

Malina, R. (2006). Weight training in youth-growth, maturation, and safety: an evidence-based review. Clinical Journal of Sport Medicine, 16(6), 478-487.

Marfell-Jones, M., Stewart, A., \& de Ridder, J. (2012). International standards for anthropometric assessment.

Maud, P., \& Foster, C. (2006). Physiological assessment of human fitness: $<\mathrm{p}>2$ nd ed. $<$ br $><$ br $>$ Champaign, IL: Human Kinetics, c2006. $<$ br $><$ br $>$ viii, 319 p.: illustrations; $29 \mathrm{~cm} .</ \mathrm{p}>$.

Microsoft, C. (2010). Excel.

Misigoj-Duraković, M., \& Heimer, S. (1992). Characteristics of the morphological and functional status of kayakers and canoeists. The Journal of sports medicine and physical fitness, 32(1), 45-50.

Onis, M., Onyango, A., Borghi, E., Siyam, A., Nishida, C., \& Siekmann, J. (2007). Development of a WHO growth reference for school-aged children and adolescents. Bulletin of the World health Organization, 85(9), 660-667.
Retamales, F. (2011). Determinación de perfil antropométrico en deportistas de la disciplina de Canotaje pertenecientes al programa gubernamental de Centro de Entrenamiento Regional (CER), de la región del Maule, Chile. Ciencias de la actividad Fisica U.C.M., 12(1), 21 -26.

Ross, W., \& Kerr, D. (1991). Fraccionamiento de la masa corporal: un nuevo método para utilizar en nutrición clínica y medicina deportiva. Apunts, 18, 175-187.

Shephard, R., Labarre, R., Jéquier, J., Lavallee, H., Rajic, M., \& Volle, M. (1985). The "unisex phantom," sexual dimorphism, and proportional growth assessment. American journal of physical anthropology, 67(4), 403-412. doi: 10.1002/ajpa.1330670412

Sosa, J. (2006). Valoración del somatotipo y proporcionalidad de futbolistas universitarios mexicanos respecto a futbolistas profesionales/. Evaluation of the somatotype and proportionality of university soccer players with respect to professional soccer players. Revista Internacional de Medicina y Ciencias de la Actividad Física y del Deporte(21), 2.

Tschiene, P. (1996). Enfoque necesario en la práctica del entrenamiento: dirigir la adaptación biológica en el entrenamiento modelo. Motricidad: revista de ciencias de la actividad fisica y del deporte(2), 9-37.

Van Someren, K., \& Howatson, G. (2008). Prediction of flatwater kayaking performance. International journal of sports physiology and performance, 3(2), 207-218.

Datos de Correspondencia:

Francisco Javier Retamales Muñoz

Lientur 1457, Concepción,

CHILE

+56998370253

Retamales.fco@gmail.com

RECIBIDO: 30-10-2015

ACEPTADO: 30-11-2015 